\title{
A Robust Blind Audio Watermarking Scheme Based on Singular Value Decomposition and Neural Networks
}

\author{
Yu YANG*, Min LEI \\ Information Security Center, Beijing University of Posts and Telecommunications \\ National Engineering Laboratory for Disaster Backup and Recovery, Beijing University of Posts and \\ Telecommunications \\ Beijing, 100876, China \\ Huaqun LIU \\ School of Information and Mechanical Engineering, Beijing Institute of Graphic Communication \\ Beijing, 102600, China \\ Yongmei CAI \\ College of Computer Science and engineering, Xinjiang University of Finance and Economic \\ Urumqi, 830012, China \\ Guoyuan LIN \\ School of Computer Science and Technology, China University of Mining and Technology \\ Xuzhou, 221116, China \\ State Key Laboratory for Novel Software Technology, Nanjing University \\ Nanjing, 211102, China
}

Received 6 April 2013

Accepted 27 May 2013

\begin{abstract}
The paper proposes a novel blind audio watermarking scheme based on singular value decomposition (SVD) and neural networks $(\mathrm{NN})$, which makes use of memorization and adaptation capabilities of NN. Experimental results show that the proposed watermarking algorithm is strongly robust against several kinds of attacks such as noise addition, re-sampling, re-quantization, MP3 compression, replace and so on. Additionally, the proposed algorithm achieves signal-to-noise radio (SNR) values ranging from $21 \mathrm{~dB}$ to $48 \mathrm{~dB}$ for different watermarked sounds and embedded strengths. These results demonstrate that the proposed watermarking method is a suitable candidate for audio copyright protection.
\end{abstract}

Keywords: Blind watermarking, singular value decomposition, neural networks, copyright protection.

\section{Introduction}

With the development of multimedia services, such as electronic commerce, pay-per-view, video-on-demand, electronic newspapers, and peer-to-peer media sharing, multimedia data can be obtained quickly over high speed network connections. However, authors, publishers, owners and providers of multimedia data are reluctant to grant the distribution of their documents in a

*Corresponding author: yangelm@vip.sina.com 
network environment because the ease of intercepting, copying and redistributing electrical data in their original forms facilitates their copyright violation.

Digital watermarking protocol has provided a method for the protection of digital images (video or audio) from unlawful copying and manipulation. Digital watermarking can be defined as the process of embedding data into a multimedia element such as image, audio or video. The embedded data can be extracted in the future from the multimedia element for various purposes, which includes copyright protection, access control and broadcast monitoring. The copyright data may be in the form of text ${ }^{1,2}$, image $e^{3-5}$, audio ${ }^{6-8}$ and video. We focus on the audio watermarking algorithm in the paper.

The type of information needed in watermarking is an important criterion in classification of different watermarking schemes:

- Non-blind watermarking scheme: requires both the original audio and secret keys for watermark embedding.

- Semi-blind watermarking scheme: requires the secret keys and the watermark itself.

- Blind watermarking scheme: only requires the secret keys.

Watermarking embedding technology can be generally classified into categories: spatial domain and frequency (transform) domain. In spatial domain, the watermark is embedded directly to the pixel locations. Frequency domain watermarking methods are based on the modification of frequency components. Spatial domain methods are less complex but are not as robust as transform domain methods against various attacks ${ }^{9}$. Most of transform domain watermarking schemes are non-blind or semi-blind.

$\mathrm{W} \mathrm{N}$ Lie and L C Chang ${ }^{10}$ proposed a blind audio watermarking system in which the embedded strength of the audio modifications was limited by the necessity to produce an output signal for watermark extraction. The watermark signals ware generated with a key, depending on the amplitude and frequency of audio signals in order to minimize the audibility of the watermarked signals. Zeng et al. ${ }^{11}$ presented a blind watermarking method that embedded watermarks into discrete cosine transform (DCT) coefficients by utilizing a quantization index modulation technique. Pooyan et al. ${ }^{12}$ proposed an audio watermarking method that embedded watermarks in a wavelet domain. The watermarked data were encrypted with a synchronization code and embedded into low frequency coefficients of the sound in the wavelet domain. The magnitude of quantization step and embedding strength was adaptively determined according to the characteristics of the human auditory system. Wang et al. ${ }^{13}$ proposed a blind audio watermarking scheme by using adaptive quantization against the synchronization attack. Moreover, the multi-resolution characteristics of discrete wavelet transform (DWT) and the energy compression characteristics of DCT were integrated into this scheme to improve the transparency of digital watermark. Watermark was then embedded into low frequency components by using adaptive quantization according to the human auditory system. Liu et al. ${ }^{14}$ introduced a blind watermarking scheme that took the advantages of the attack-invariant feature of the cepstrum domain and the error-correction capability of $\mathrm{BCH}$ code to increase the robustness as well as imperceptibility of audio watermarking.

Those blind watermark schemes were built on the principle of spread spectrum. Although the method allows for reliable communication even for strong attacks, spread spectrum based system offer relatively little robustness when the host signal is not known at the decoder and blind detection of spread spectrum watermark suffers significantly from host data interference. What's more, the robustness of the currently watermarking schemes is far from satisfaction, especial for the audio watermarking scheme. Most of them can resist against only certain limited attacks.

In order to avoid the above two disadvantages, the paper proposes a novel blind audio watermarking scheme based on singular value decomposition (SVD) and neural networks (NN). The proposed scheme can embed the watermarking information in optimal location which can get from $\mathrm{NN}$ technology, but the traditional methods embed the watermarking information in random continuous location. At the same time, because neural networks possess memorization and adaptation capabilities, the proposed scheme can extract watermarking information without original audio.

The paper is organized as follows. Section 2 introduces basic concepts for neural networks. Section 3 describes our watermark-embedding and watermarkextraction algorithms. Section 4 shows experimental results illustrating that the proposed method can achieve a good balance between imperceptibility and robustness. A brief conclusion is presented in Section 5. 


\section{Neural Networks}

An artificial neural network (ANN) is a mathematical or computational model that is inspired by the structure and/or functional aspects of biological neural networks. A neural network (NN) consists of an interconnected group of artificial neurons, and it processes information of a connectionist approach to computation. In most cases an ANN is an adaptive system that changes its structure based on external or internal information that flows through the network during the learning phase. Modern neural networks are non-linear statistical data modeling tools. They are usually used to model complex relationships between inputs and outputs or to find patterns in data.

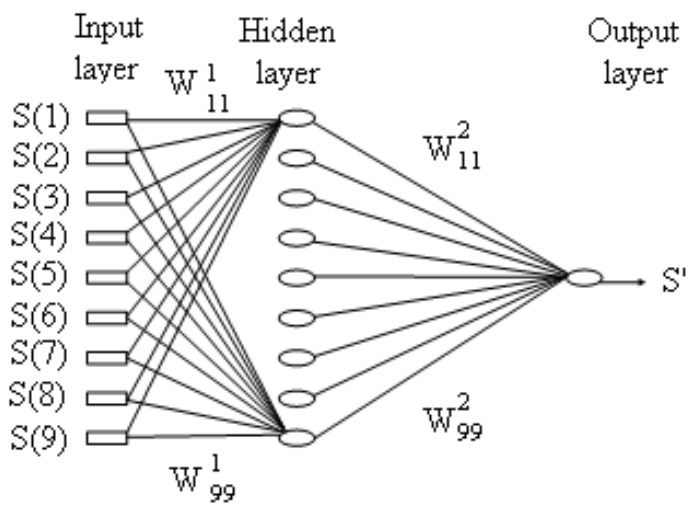

Fig. 1. The structure of a neural network

The fig. 1 shows the architecture of $\mathrm{NN}$, which is a 9-9-1 multiplayer perception. Namely, the NN comprises an input layer with 9 nodes, a hidden layer with 9 nodes, and an output layer with a single node ${ }^{15}$. In addition, the back propagation algorithm is adopted for training $\mathrm{NN}$ over a set of training patterns $\gamma$ that is specified by

$$
\gamma=\left\{\left(I_{i}, O_{i}\right) \mid i=1,2, \ldots, M_{1} \times M_{2}\right\}
$$

where $I_{i}=\left(s_{i}(1), s_{i}(2), s_{i}(3), s_{i}(4), s_{i}(5), s_{i}(6), s_{i}(7)\right.$, $\left.s_{i}(8), s_{i}(9)\right)$ is the input, $O_{i}$ is the expected output and $M_{1} \times M_{2}$ is the size of watermark.

When the training process for $\mathrm{NN}$ is completed, a set of synaptic weights $t$, characterizing the behavior of the trained neural network (TNN), can be obtained and represented by:

$$
W^{\prime}=\left\{\begin{array}{l|l}
W_{u v}^{1} & \begin{array}{l}
u=1,2, \ldots, 9 \\
v=1,2, \ldots, 9
\end{array}
\end{array}\right\}\left\{\begin{array}{l|l}
W_{u v}^{2} & \begin{array}{l}
u=1, \\
v=1,2, \ldots, 9
\end{array}
\end{array}\right\}
$$

Accordingly, TNN performs a mapping from the space in which $I_{i}$ is defined to the space in which $O_{i}$ is defined. In other words, TNN can memorize the relationship between the original audio and watermarked audio.

\section{Proposed Blind Watermarking Scheme}

In general, the watermarking scheme involves watermarking generation, watermarking embedding algorithm and watermark exaction algorithm. Suppose that the watermark information is a binary image $W(i, j),\left(0 \leq i<M_{1}, 0 \leq j<M_{2}\right)$ that contain $M_{1} \times M_{2}$ bits, where $W(i, j) \in\{0,1\}$. Now we will explain the watermarking embedding algorithm and watermark exaction algorithm in detailed.

\subsection{Watermarking Embedding Algorithm}

Let $X=\left(x_{1}, x_{2}, \ldots, x_{M}\right)$ denote the host audio, $W(i, j),\left(0 \leq i<M_{1}, 0 \leq j<M_{2}\right) \quad$ denote the watermarking information. The detailed watermark embedding algorithm is given in the following steps (Fig.2).

Step 1: Dimension reduction of watermark image. Because the audio signal is one-dimensional and watermarking $W(i, j)$ is two-dimensional. Therefore the watermarking should be reduced into onedimensional signal:

$$
\begin{aligned}
& w=\left\{w(i)=w\left(m_{1}, m_{2}\right) \mid 1 \leq m_{1} \leq M_{1}, 1 \leq m_{2}\right. \\
& \left.\leq M_{2}, 1 \leq i \leq M_{1} \times M_{2}\right\}
\end{aligned}
$$

Step 2: Choose the optimal audio blocks. Let an audio $X=\left(x_{1}, x_{2}, \ldots, x_{M}\right)$ with M PCM (pulse-code modulation) samples be segmented into $N=\lfloor M / 1600\rfloor$ blocks $B=\left\{b_{1}, b_{2}, \ldots, b_{N}\right\}$, where $N \geq M_{1} \times M_{2}$. Each block includes 1600 samples. We can choose $M_{1} \times M_{2}$ blocks from N blocks, which can be embed into one bit watermark information for each block. The choosing algorithm is as following:

(1) The 2-level DWT is applied to the audio block $b_{i}(i=1,2, \ldots, N)$, and then we select the approximate sub-band coefficients $C a 1$, which has 400 samples.

(2) The approximate sub-band coefficients Cal is decomposed on DCT, and the first quarter of the DCT coefficients is converted into a matrix $A_{i}(i=1,2, \ldots$, $N)$ with $10 \times 10$.

(3) The singular value decomposition is applied to the matrix $A_{i}(i=1,2, \ldots, N)$, then can get a diagonal 
matrix $S_{i}(i=1,2, \ldots, N)$ with $10 \times 10$.

(4) Select $S_{k}\left(k \in K=\left\{j_{1}, j_{2}, \ldots, j_{M_{1} \times M_{2}}\right\}, j_{i} \in\{1,2, \ldots, N\}\right.$, $\left.i=\left\{1,2, \ldots, M_{1} \times M_{2}\right\}\right)$, which satisfies $S_{k}(1,1) \geq$ $S_{n}(1,1)$, where $k \in K, n \in\{1,2, \ldots N\} / K$.

Step 3: The marked elements of diagonal matrix $S_{j_{k}}$ ' $(1,1)$ are obtained by using the following equation: $S_{j_{k}}{ }^{\prime}(1,1)=S_{j_{k}}(1,1)+\alpha w_{k}, j_{k} \in K$, where the embedded strength $\alpha=0.2$ and $w_{k}$ is the watermark.

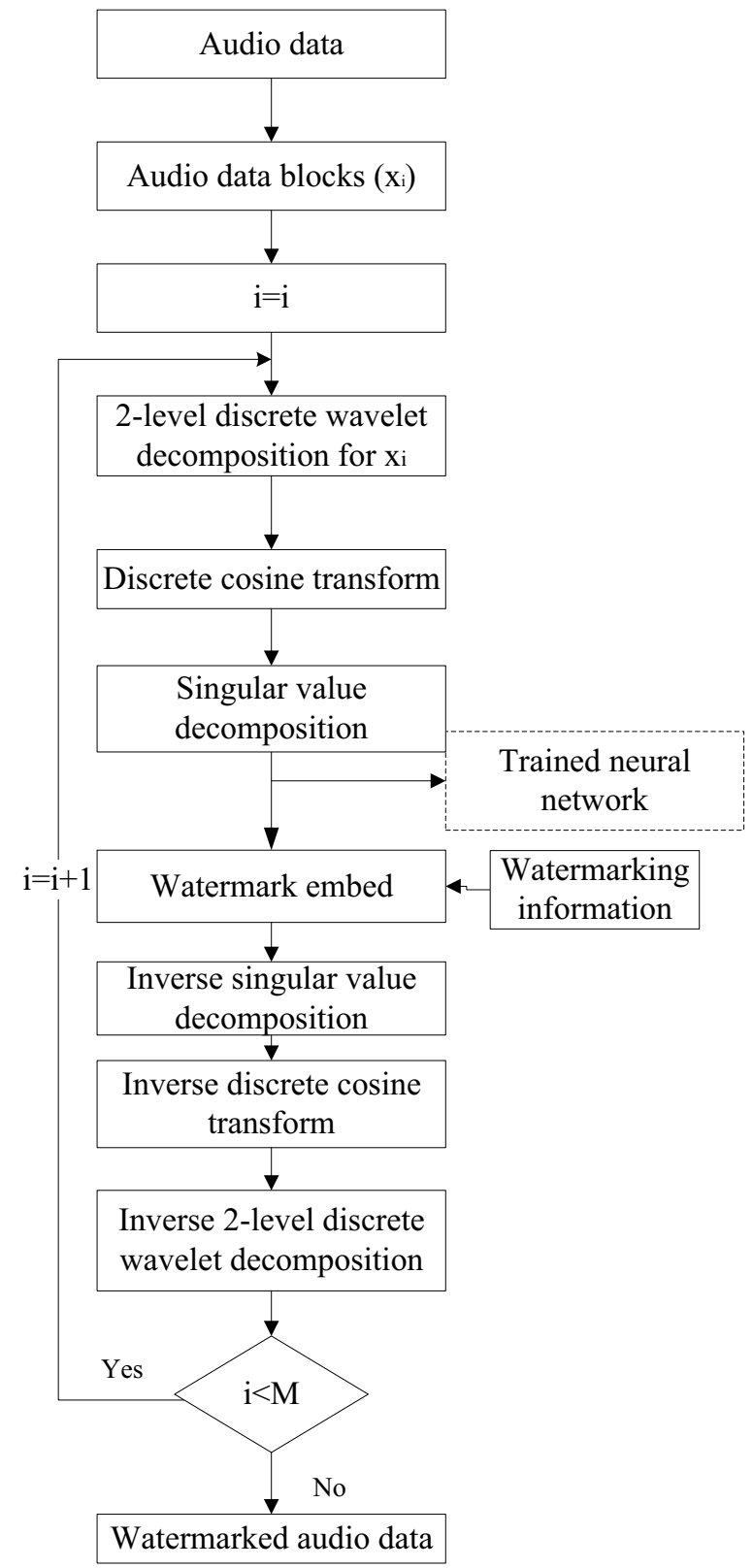

Fig. 2. The structure of watermark embedding
Step 4: Inverse singular value decomposition is applied to the modified diagonal matrix, and gets a matrix $S^{\prime}{ }_{j_{k}}$.

Step 5: The matrix $S_{j_{k}}^{\prime}$ reduces into onedimensional signal, and gets the modified DCT coefficients. Then the inverse discrete cosine transform is applied to the modified DCT coefficients, and gets the modified DWT coefficients.

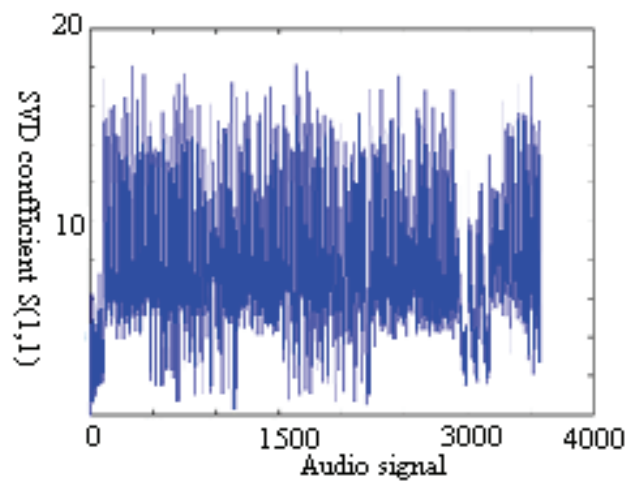

(a) Pop

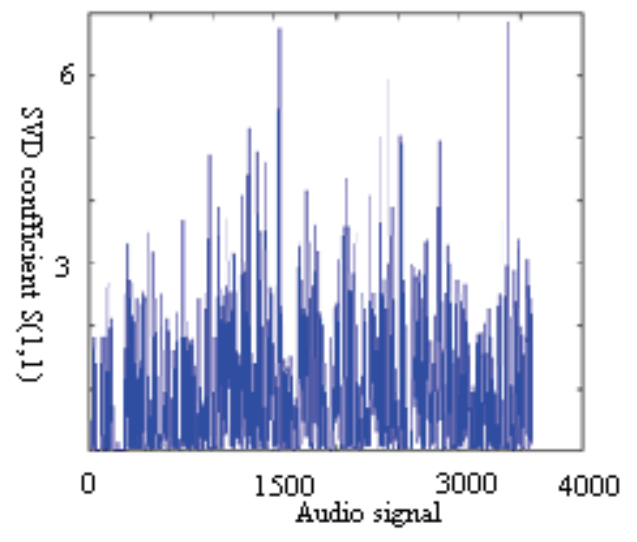

(b) Speech

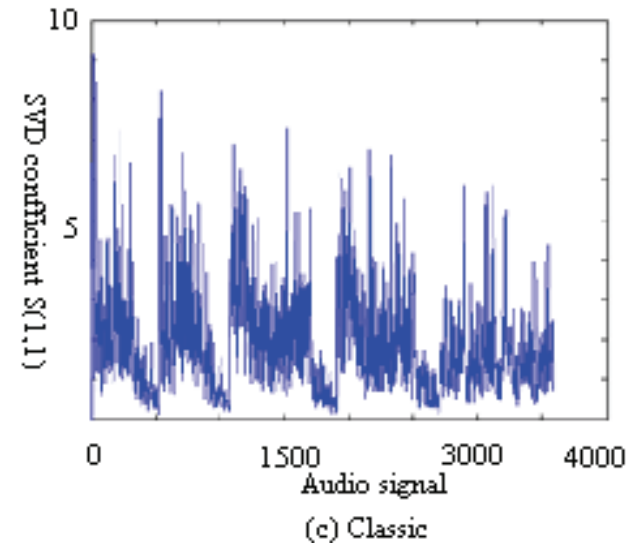

Fig. 3. SVD coefficient $\mathrm{S}(1,1)$ of audio 
Step 6: The inverse 2-level discrete wavelet packet decomposition is applied to the modified DWT coefficients to transform them back to the audio signal in time domain.

Step 7: We can compute the difference $W^{\prime}$ between the original audio and the embedded watermark audio by using the trained neural network, and get a map which the input is $\left(S_{j_{k}-t}, \ldots S_{j_{k}-1}, S_{j_{k}}^{\prime}, S_{j_{k}+1}, \ldots, S_{j_{k}+t}\right)$ and the expected output is $S_{j_{k}}$.

Most of currently watermark embedding algorithms are embedded into one bit watermarking information in turn $^{[12-14,16-18]}$, but our proposed algorithm finds the optimal frame using the TNN technology (figure 2), and then embeds into the watermarking information. The SVD coefficients $\mathrm{S}(1,1)$ of the audio signal is not collected in random continuous audio signal as show in Fig. 3.

\subsection{Watermark Extraction Algorithm}

TNN obtained from the watermark embedding, can memorize the relationships between an original audio and the corresponding watermarked audio. Listed below are the parameters which are required in the watermark extraction.

- All synaptic weights of the TNN, $W^{\prime}$.

- The number $M_{1} \times M_{2}$ of the bits of the watermark $W$.

The structure of the watermark extraction is depicted in Fig. 4. The detailed watermark extraction algorithm is given in the following steps.

Step 1: Let an audio $X w=\left(x w_{1}, x w_{2}, \ldots, x w_{M}\right)$ be segmented into $N=\lfloor M / 1600\rfloor$ blocks $B w=\left\{b w_{1}, b w_{2}, \ldots, b w_{N}\right\}$, where $N \geq M_{1} \times M_{2}$. Each block includes 1600 samples. We can choose $M_{1} \times M_{2}$ blocks from $\mathrm{N}$ blocks, which can be embed into one bit watermarking information for each block.

Step 2: The 2-level discrete wavelet packet decomposition is applied to the watermarked audio block $b w_{j_{k}}\left(k=1,2, \ldots, M_{1} \times M_{2}\right)$, then select the approximate sub-band coefficients $\mathrm{Calw}$, which has 400 samples.

Step 3: The approximate sub-band coefficients Ca1w is decomposed on DCT, and the first quarter of the DCT coefficients is converted into a matrix $A w_{j_{k}}\left(k=1,2, \ldots, M_{1} \times M_{2}\right)$ with $10 \times 10$.

Step 4: The singular value decomposition is applied to the matrix $A w_{j_{k}}\left(k=1,2, \ldots, M_{1} \times M_{2}\right)$, then can get a diagonal matrix $S w_{j_{k}}\left(k=1,2, \ldots, M_{1} \times M_{2}\right)$ with $10 \times 10$.

Step 5: We can get the physical output $S w_{j_{k}}^{\prime}$ according to TNN which the input is:

$$
\begin{aligned}
& \left(S w_{j_{k}-4}(1,1), S w_{j_{k}-3}(1,1), S w_{j_{k}-2}(1,1), S w_{j_{k}-1}(1,1), S w_{j_{k}}(1,1),\right. \\
& \left.S w_{j_{k}+1}(1,1), S w_{j_{k}+2}(1,1), S w_{j_{k}+3}(1,1), S w_{j_{k}+4}(1,1),\right)
\end{aligned}
$$

Step 6: According to the watermarked simple $S w_{j_{k}}(1,1),\left(k=1,2, \ldots, M_{1} \times M_{2}\right)$ and corresponding physical $S w_{j_{k}}^{\prime}$ for TNN, the $k$-th bit of the extracted watermark $\mathrm{W}$ can be estimated by:

$$
W_{k}=\left\{\begin{array}{c}
1, i f S w_{j_{k}}(1,1)-S w_{j_{k}}{ }^{\prime}>0.05 \\
0, \text { else }
\end{array}\right.
$$

Step 7: We can get the binary image according to the extracted watermark.

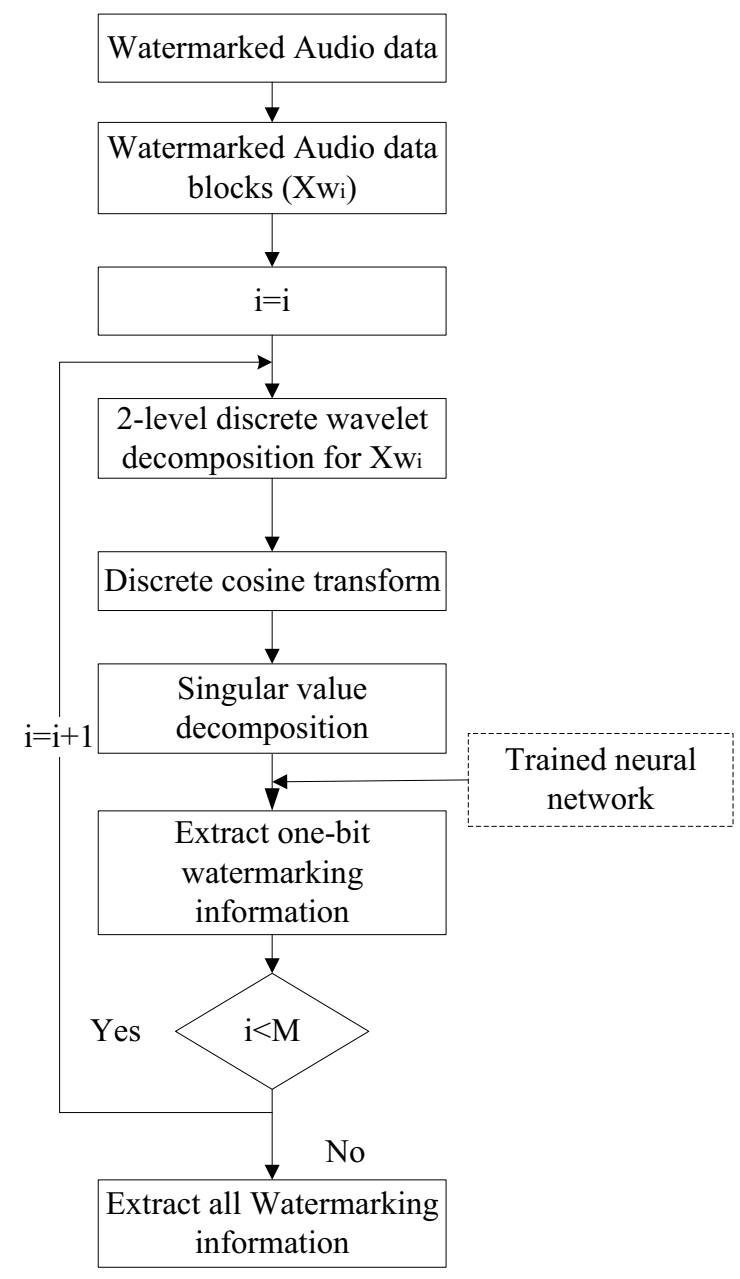

Fig.4. Structure of watermark extraction 


\section{Experimental Results and Analysis}

\subsection{Experiment Setting and Evaluation Metrics}

In this experiment, two binary stamp image with size $32 \times 32$ (i.e., $M_{1}=M_{2}=32$ ), displayed in Fig. 5, are taken as the original watermark And we evaluate the performance of our proposed watermarking method for different types of 16 bits mono audio signals sampled at 44.1 KHz as shown in Fig. 6(a), (c), (e). The sound files are: (a) Pop, (c) Speech, (e) Classic. Each audio file contains 5,741,998 samples with duration of 130 seconds. The watermarked audio is shown as Fig. 6(b), (d), (f).

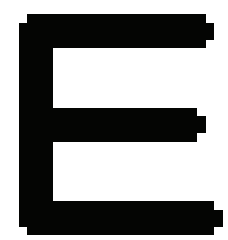

Fig. 5. Watermarks with size $32 \times 32$

In order to evaluate the quality of watermarked audio, the following signal-to-noise radio (SNR) equation is used:

$$
S N R=10 \log _{10} \frac{\sum_{n=1}^{N} Y^{2}(n)}{\sum_{n=1}^{N}\left[Y(n)-Y^{\prime}(n)\right]^{2}}
$$

where $Y(n)$ and $Y^{\prime}(n)$ are original audio signal and watermarked audio signal respectively.

In order to evaluate the robustness of the watermarked algorithm, the following normalized coefficient (NC) and bit error rate (BER) are employed respectively:

$$
\begin{gathered}
N C\left(W, W^{*}\right)=\frac{\sum_{i=1}^{M_{1}} \sum_{j=1}^{M_{2}} W(i, j) W^{*}(i, j)}{\sqrt{\sum_{i=1}^{M_{1}} \sum_{j=1}^{M_{2}} W(i, j)^{2}} \times \sqrt{\sum_{i=1}^{M_{1}} \sum_{j=1}^{M_{2}} W^{*}(i, j)^{2}}} \\
B E R=\left(\sum_{i=1}^{M_{1}} \sum_{j=1}^{M_{2}}\left|W(i, j)-W^{*}(i, j)\right|\right) /\left(M_{1} \times M_{2}\right)
\end{gathered}
$$$$
\text { where } W(i, j) \text { and } W^{*}(i, j) \text { are the original }
$$$$
\text { watermark and extract watermark respectively, and }
$$
$M_{1} \times M_{2}$ is the size of watermark.

\subsection{Imperceptibility Test and Analysis}

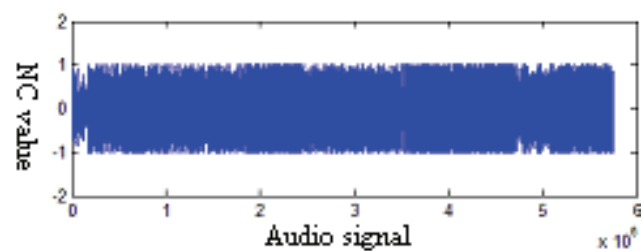

(a)

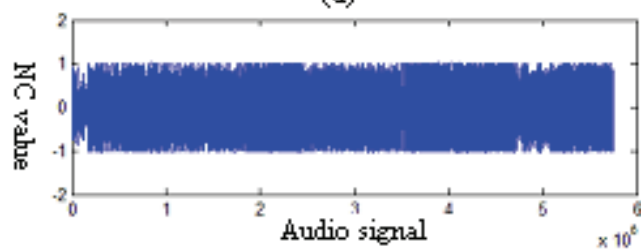

(b)

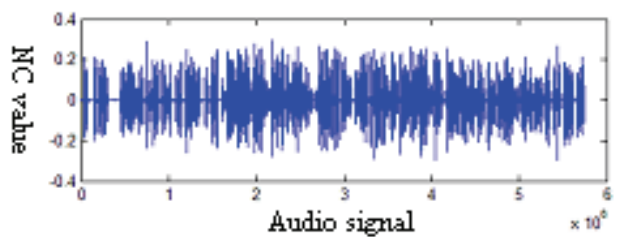

(c)

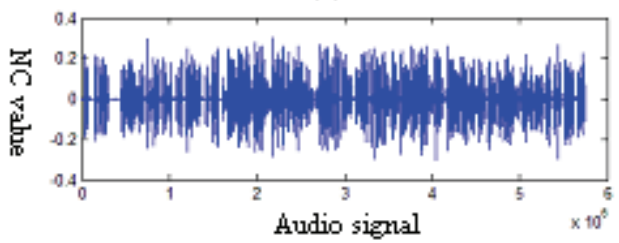

(d)

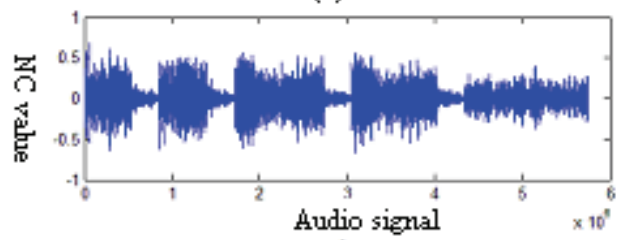

(e)

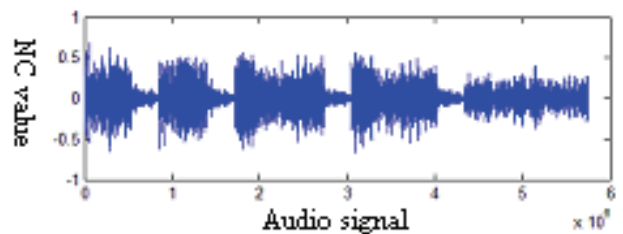

(f)

Fig. 6. Original audio and watermarked audio for three kinds of sound

According to the proposed watermarking scheme, we can conclude that informal listening by using head set reveals that the watermark embedded into the original audio signal, which does not affect the quality of the sound and ensures the imperceptibility of the embedded watermark.

To observe Fig. 6, these three watermarked audio are almost similar to their original versions. The difference between the original audio and watermarked 
audio is showed as Fig. 7, where $X$ denote the audio signal, $X^{\prime}$ denotes the watermarked audio signal. Therefore, the proposed method remarkably possesses imperceptible capability for making watermarks inaudible.

Table 1 shows the SNR results of the proposed watermarking method for different values of embedded strength $\alpha$ and different types of audio. Our proposed method achieves SNR values ranging from $21 \mathrm{~dB}$ to $48 \mathrm{~dB}$ for different watermarked sounds.

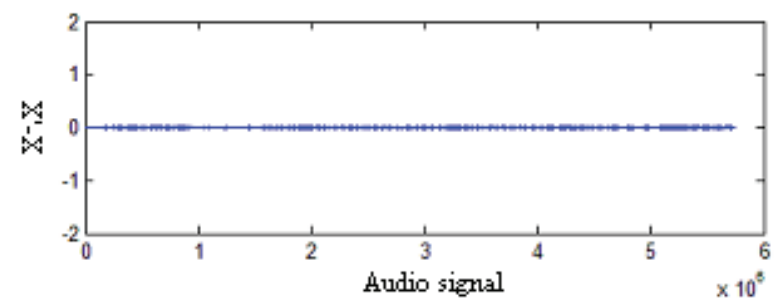

(a) Pop

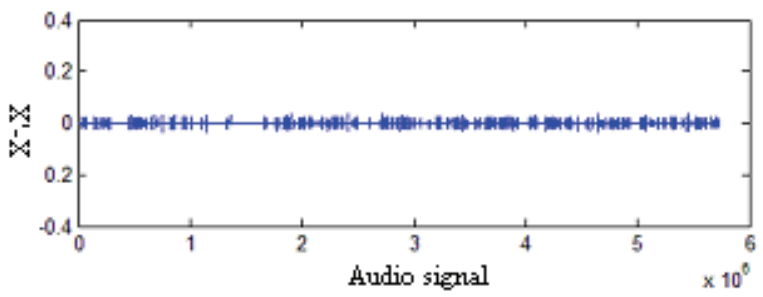

(b) Speech

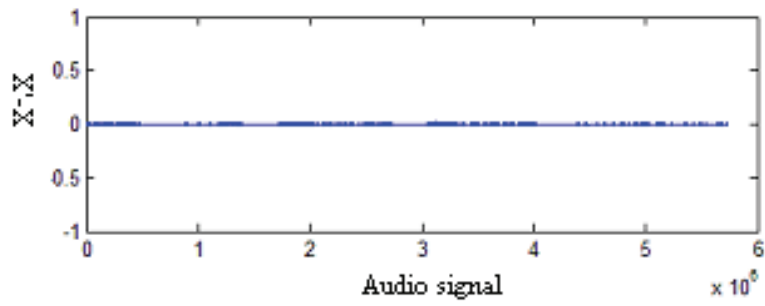

(c) Classic

Fig. 7. The difference signal between the original audio and watermarked audio

Table 1. SNR results of the proposed watermarking method

\begin{tabular}{|c|c|c|c|}
\hline & Pop & Speech & Classic \\
\hline$\alpha=0.1$ & 48.406 & 31.386 & 37.186 \\
\hline$\alpha=0.2$ & 42.385 & 25.366 & 31.166 \\
\hline$\alpha=0.3$ & 38.864 & 21.844 & 27.644 \\
\hline
\end{tabular}

\subsection{Robustness Test and Analysis}

In order to test the robustness of our proposed method, eight different types of attacks are as follows:
(1) Noise addition: $20 \mathrm{~dB}$ additive white Gaussian noise (AWGN) is added to the watermarked audio signal

(2) Re-sampling: The watermarked signal originally sampled at $44.1 \mathrm{kHz}$ is re-sampled at $22.050 \mathrm{kHz}$, and then restored by sampling again at $44.1 \mathrm{kHz}$.

(3) Low-pass filtering: $11.025 \mathrm{kKz}$ for the low-pass filter cutoff frequency.

(4) Re-quantization: the 16 bit watermarked audio signal is quantized down to 8 bits/sample and again re-quantized back to 16 bits/sample.

(5) MP3 compression: MPEG-1 layer 3 compression with $64 \mathrm{kbps}$ is applied to the watermarked signal.

(6) MP3 compression: MPEG-1 layer 3 compression with $32 \mathrm{kbps}$ is applied to the watermarked signal.

(7) MP3 compression: MPEG-1 layer 3 compression with $128 \mathrm{kbps}$ is applied to the watermarked signal.

(8) Replace.

Table 2 shows the NC and BER of the proposed watermarking method in terms of robustness against several kinds of attacks applied to three different types of watermarked audio signal "Speech", "Classic" and "Pop" respectively. We can see the third attack is weak robust compared to other attacks in the proposed algorithm. It depends on the characteristic of audio.

Table 2. The result of attacks

\begin{tabular}{|c|c|c|c|c|c|c|}
\hline \multirow{2}{*}{ Result } & \multicolumn{2}{|c|}{ Speech } & \multicolumn{2}{c|}{ Classic } & \multicolumn{2}{c|}{ Pop } \\
\cline { 2 - 7 } Attack & NC & BER & NC & BER & NC & BER \\
\hline 1 & 1 & 0 & 0.999 & 0.001 & 1 & 0 \\
\hline 2 & 1 & 0 & 1 & 0 & 1 & 0 \\
\hline 3 & 0.992 & 0.008 & 0.986 & 0.015 & 0.988 & 0.011 \\
\hline 4 & 1 & 0 & 1 & 0 & 1 & 0 \\
\hline 5 & 1 & 0 & 1 & 0 & 1 & 0 \\
\hline 6 & 1 & 0 & 1 & 0 & 1 & 0 \\
\hline 7 & 1 & 0 & 1 & 0 & 1 & 0 \\
\hline 8 & 1 & 0 & 1 & 0 & 1 & 0 \\
\hline
\end{tabular}

Overall, our proposed watermarking method provides good results in terms of robustness against several attacks such as noise addition, re-sampling, requantization, MP3 compression, and replace as well as provides good SNR values for different watermarked sounds. 
Table 3 compares the proposed algorithm and other algorithms, we can easily get the robustness of the proposed algorithm is stronger than other algorithms.

Table 3 Comparison between our algorithm and other algorithm

\begin{tabular}{|c|c|c|c|c|c|}
\hline $\begin{array}{c}\text { Att } \\
\text { ack }\end{array}$ & $\begin{array}{c}\text { BER } \\
(\text { our })\end{array}$ & $\begin{array}{c}\text { BER } \\
{[19]}\end{array}$ & $\begin{array}{c}\text { BER } \\
{[20]}\end{array}$ & $\begin{array}{c}\text { BER } \\
{[21]}\end{array}$ & $\begin{array}{c}\text { BER } \\
{[22]}\end{array}$ \\
\hline 1 & 0 & 2.34 & 0 & 5.13 & 4.98 \\
$(20 \mathrm{db})$ & $10 \mathrm{~dB}$ & & & \\
\hline 2 & 0 & 2.03 & 1 & 13.64 & 0 \\
& $(22.05$ & $(22.05$ & $(22.05$ & $(22.05$ & $(22.05$ \\
& $\mathrm{kHz})$ & $\mathrm{kHz})$ & $\mathrm{kHz})$ & $\mathrm{kHz})$ & $\mathrm{kHz})$ \\
\hline 3 & 0.15 & 2.19 & 0 & 18.06 & \\
& $(11.025$ & $(8$ & $(11.025$ & $(11.025$ & - \\
& $\mathrm{kHz})$ & $\mathrm{kHz})$ & $\mathrm{kHz})$ & $\mathrm{kHz})$ & \\
\hline 6 & 0 & 4.38 & 2 & 5.71 & 24.18 \\
& $(32$ & $(64$ & $(32$ & $(128$ & $(32$ \\
& $\mathrm{kbps})$ & $\mathrm{kbps})$ & $\mathrm{kbps})$ & $\mathrm{kbps})$ & $\mathrm{kbps})$ \\
\hline
\end{tabular}

\section{Conclusion}

In this paper, neural network techniques are successfully incorporated into audio watermarking to develop a novel blind watermarking method. Because of TNN's memorization and adaptation capabilities, the algorithm can extract watermarks without original audio, which is required by all other proposed methods audio for watermark extraction. Experimental results indicate that our proposed watermarking algorithm has strong robustness against several kinds of attacks such as noise addition, re-sampling, re-quantization, MP3 compression, and replace. Additionally, the proposed algorithm achieves SNR values ranging from $21 \mathrm{~dB}$ to $48 \mathrm{~dB}$ for different watermarked sounds and different embedded strengths. These results demonstrate that the proposed algorithm is a suitable candidate for audio copyright protection.

\section{Acknowledgements}

This work is supported by the National Natural Science Foundation of China under Grant NO 60973146, NO 61170269, NO 61003285, NO 61170272, and the Fundamental Research Funds for the Central Universities under Grant No. BUPT2013RC0308,

\section{BUPT2013RC0311.}

\section{Reference}

1 H M Meral, E Sevinc, E Unkar, B Sankur, A S Ozsoy and $\mathrm{T}$ Gungor, Syntactic tool for text watermarking, in
Proc. of the SPIE on Security, Steganography and Watermarking of Multimedia Contents, eds. E J Delp and P W Wong. (San Jose, CA, 2007), pp. 149-151.

2 Y. Liu, X. Sun and Y. Wu, A natural language watermarking based on Chinese syntax, J. Advances in Natural Computation, (Springer, 2005), pp. 958-961.

3 K V Swamy, B C Mohan, Y V B Reddy and S. S Kumar, Image compression and watermarking scheme using scalar quantization, The International Journal of Next Generation Network. 2(1) (2010) 37-47.

4 B Chandra Mohan and S Srinivas Kumar, Robust multiple image watermarking scheme using discrete cosine transform with multiple descriptions, International Journal of Computer Theory and Engineering, 5(1) (2009) 527-532.

5 Y. Q. Chen, Y Q Zhang, H P Hu and H F Ling, A novel gray image watermarking scheme, Journal of Software, 5(6) (2011) 849-856.

6 P K Dhar, M I Khan and S Ahmad, A new DCT-based watermarking method for copyright protection of digital audio, International Journal of Computer Science \& Information Technology, 5(2) (2010) 91-101.

7 S Lagzian, M Soryani and M Fathy, A new robust watermarking scheme based on RDWT-SVD, International Journal of Intelligent Information Processing, 1(2) (2011) 22-29.

$8 \mathrm{H}$ H Tsai, Audio watermarking based on HAS and neural networks in DCT domain, EURASIP Journal on Applied Signal Processing, 3 (2003) 252-263.

9 L. Liang and S. Qi, A new SVD-DWT composite watermarking, in Proc. of IEEE Int. Conf. on Signal Processing, eds. X Z Wei (Beijing, China, 2006), pp. 365-372.

10 V P. Bassia, I. Pitas and N. Nikolaidis, Robust audio watermarking in the time domain," $J$. IEEE Transaction on Multimedia, 2(3) (2001) 232-241.

11 G. Zeng and Z. Qiu, Audio watermarking in DCT: embedding strategy and algorithm, in Proc. of 9th Int. Conf. on Signal Processing, eds. L A Zadeh and J. Kacprzyk (Beijing, China, 2008), pp. 2193-2196.

12 M. Pooyan and A. Delforouzi, Adaptive and robust audio watermarking in wavelet domain, in Proc. of Int. Conf. on International Information Hiding and Multimedia Signal Processing, eds, B Y Liao, J S Pan, L C Jain, M Liao, H Noda and A T S Ho (Kaohsiung, Taiwan, 2007), pp. 287-290.

13 X. Y. Wang and H. Zhao, A novel synchronization invariant audio watermarking scheme based on DWT and DCT, J. IEEE Transaction on signal Processing, 12(54) (2006) 4835-4840.

14 S. C. Liu and S. D. Lin, BCH code based robust audio watermarking in the cepstrum domain, Journal of Information Science and Engineering, 22 (2006) 535543.

15 S. Haykin, Neural networks: a comprehensive foundation, 1st edn. (Macmillan College Publishing Company, New York, NY, USA, 1995). 
16 Chih-chin Lai and Cheng-chih Tsai, Digital image watermarking using discrete wavelet transform and singular value decomposition, IEEE transactions on instrumental and measurement, 11(59), (2010) 30603063.

17 A. Hanaa Abdallah, M. Mohiy Hadhoud and A. Abdalhameed Shaalan, SVD-based watermarking scheme in complex wavelet domain for color video, in Proc. of Int. Conf. on Computer Engineering \& Systems, eds, Gamal Aly, Hani Mahdi, Ashraf Salem, M. Watheq EI-Kharashi, Ayman Bahaa EI-Din, Mohamad A. Sobh and Mohamad Taher (Cairo, Egypt, 2009) 455 - 460.

18 Hongqin Shi, Fangliang Lv, A blind digital watermark technique for color image based on integer wavelet transform, in Proc. of Int. Conf. on Biomedical Engineering and Computer Science, eds, V. E. Muchin and Zhengbing $\mathrm{Hu}$ (Wuhan, China, 2010) 1-4.

19 Zezula R and Misurec J, Audio digital watermarking algorithm based on SVD in MCLT domain, in Proc. of Int. Conf. on Systems, eds, Ekaterina Prasolova-Forland and Manuela Popescu (Cancun, Mexico, 2008) 140-143.

20 Vivekananda B K, Indranil S and Abhijit D, An audio watermarking scheme using singular value decomposition and dither-modulation quantization. Multimedia Tools and Applications, 52(2-3), (2010) 369 $-383$.

21 R Wang, D Xu, J Chen and C. Du C, Digital audio watermarking algorithm based on linear predictive coding in wavelet domain. In Prof. of 7th Int. Conf. on Signal Processing, eds, Yuan Baozong, Ruan Qiuqi and Tang xiaofang (Beijing, china, 2004) 2393 - 2396.

22 S Wu S, J Huang J, D Huang and Y Q, Efficiently SelfSynchronized Audio Watermarking for Assured Audio Data Transmission. IEEE Trans Broadcast, 51(1), (2005) $69-76$. 